\title{
MyBP-C: one protein to govern them all
}

\author{
L. W. H. J. Heling ${ }^{1}$ - M. A. Geeves ${ }^{1} \cdot$ N. M. Kad ${ }^{1}$ (]
}

Received: 22 October 2019 / Accepted: 29 November 2019 / Published online: 20 January 2020

(C) The Author(s) 2020

\begin{abstract}
The heart is an extraordinarily versatile pump, finely tuned to respond to a multitude of demands. Given the heart pumps without rest for decades its efficiency is particularly relevant. Although many proteins in the heart are essential for viability, the non-essential components can attract numerous mutations which can cause disease, possibly through alterations in pumping efficiency. Of these, myosin binding protein $\mathrm{C}$ is strongly over-represented with $\sim 40 \%$ of all known mutations in hypertrophic cardiomyopathy. Therefore, a complete understanding of its molecular function in the cardiac sarcomere is warranted. In this review, we revisit contemporary and classical literature to clarify both the current standing of this fast-moving field and frame future unresolved questions. To date, much effort has been directed at understanding MyBP-C function on either thick or thin filaments. Here we aim to focus questions on how MyBP-C functions at a molecular level in the context of both the thick and thin filaments together. A concept that emerges is MyBP-C acts to govern interactions on two levels; controlling myosin access to the thin filament by sequestration on the thick filament, and controlling the activation state and access of myosin to its binding sites on the thin filament. Such affects are achieved through directed interactions mediated by phosphorylation (of MyBP-C and other sarcomeric components) and calcium.
\end{abstract}

Keywords cMyBP-C $\cdot$ Muscle contraction $\cdot$ Contractility $\cdot$ Cardiac $\cdot$ Myosin binding protein $\mathrm{C}$

\section{Introduction}

Muscle contraction and relaxation on the molecular level is achieved by the sliding movement of interdigitating thick filaments containing myosin and thin filaments containing actin in the sarcomere. Fundamentally, this process is driven by the cyclic interaction between myosin heads and actin filaments coupled with ATP hydrolysis and conformational changes of the myosin head (Geeves and Holmes 1999). Aside from actin and myosin the sarcomere contains an array of additional proteins that aid in the assembly or integrity of the sarcomere, and regulate the force, rate and timing of contraction.

N. M. Kad

n.kad@kent.ac.uk

1 School of Biosciences, University of Kent, Canterbury CT2 7NH, UK

\section{Structure/localisation}

Myosin binding protein C (MyBP-C) is a sarcomeric accessory protein that was first identified as a contaminant of crude skeletal muscle preparation (Starr and Offer 1971). The protein is approximately $40 \mathrm{~nm}$ in length, $3 \mathrm{~nm}$ in width, and has a molecular weight of $\sim 140 \mathrm{kDa}$ (Hartzell and Sale 1985). There are three paralogs, encoded by three different genes on different chromosomes (Fig. 1). Slow skeletal (ss)MyBP-C is encoded by $M Y B P C l$ on chromosome 12, fast skeletal (fs)MyBP-C by $M Y B P C 2$ on chromosome 19 and cardiac (c)MyBP-C by MYBPC3 on chromosome 11 . cMyBP-C was discovered after ssMyBP-C and fsMyBP-C (Hartzell and Titus 1982; Yamamoto and Moos 1983), and as the name suggests is exclusive to cardiac muscle.

The three paralogs have likely arisen through gene duplication and have similar primary structures; cardiac shares $54.4 \%$ sequence identity with fast skeletal, and for slow skeletal the identity to cardiac is $52.4 \%$ (Weber et al. 1993; Yasuda et al. 1995). The proteins are primarily formed of a series of globular domains of the immunoglobulin (Ig) or fibronectin-III (Fn3) families named C1-C10 from the $\mathrm{N}$-terminus with an additional motif or $\mathrm{M}$-domain linking 


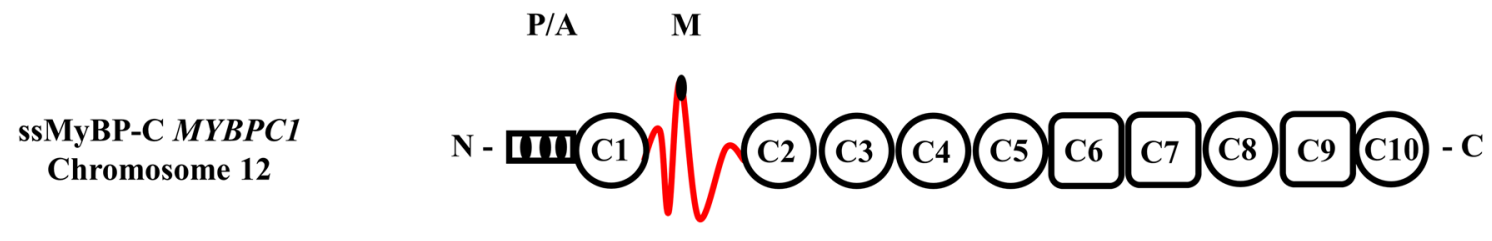

fsMyBP-C $M Y B P C 2$

Chromosome 19
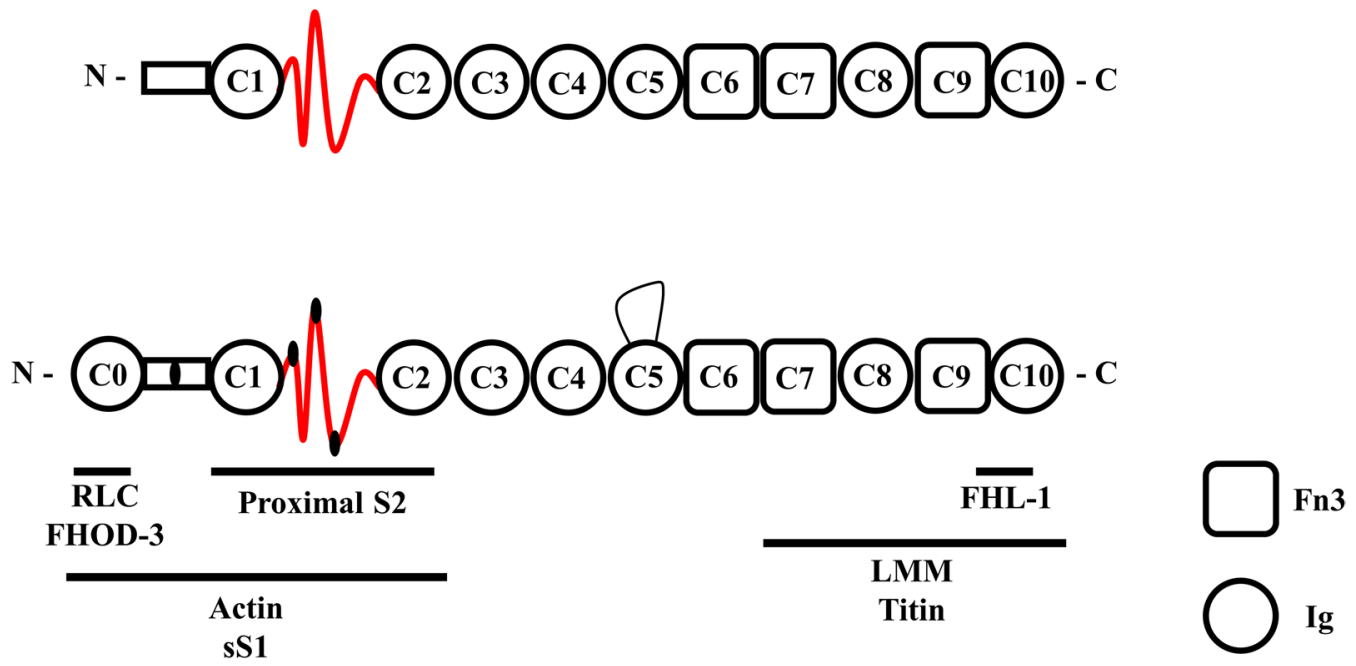

Fig. 1 Schematic diagram of full length slow skeletal (ss), fast skeletal (fs) and cardiac (c) MyBPC paralogs. Each isoform comprises three Fn3 domains and seven or eight Ig domains. The known binding partners and positions are indicated by the horizontal stripes

$\mathrm{C} 1$ and $\mathrm{C} 2$ and a proline alanine rich region (P/A) at the $\mathrm{N}$-terminus (Fig. 1). cMyBP-C has an additional Ig domain $\mathrm{C} 0$ at the $\mathrm{N}$-terminus and has 4 serine residues that can be phosphorylated in the M-domain (Yasuda et al. 1995) as well as an additional 28 amino acid loop in the C5 domain (Flashman et al. 2004).

Two studies in 1995 linked mutations on $M Y B P C 3$ to familial hypertrophic cardiomyopathy (HCM) (Bonne et al. 1995; Watkins et al. 1995), a disease that affects 1 in 200 people and the most common cause of sudden death in young people (Harvey and Leinwand 2011; Maron and Maron 2013; Semsarian et al. 2015). Currently, $40 \%$ of the sarcomeric mutations known to be linked to HCM have been found in cMyBP-C (Carrier et al. 2015). The gradual emergence of the link between cMyBP-C and HCM has shifted focus towards understanding the structure and function of cMyBP-C in disease and normal physiology. This protein is not essential for viability, confirmed in mouse knockout studies, however, significant deficits in contraction were observed, indicating a modulatory role in contraction (Harris et al. 2002). We outline some of the ways this could be achieved below. MyBP-C's other potential roles range from physiological to structural. Several below. Note the phosphorylation sites in the P/A and M domain of the ssMyBP-C and cMyBP-C paralogs are indicated by small black ellipses. The cMyBP-C has an additional 28 amino acid loop in the C5 domain

reports have described MyBP-C binding partners in the sarcomere that may offer a contribution to their formation, maintenance and general function. Calmodulin has been reported to interact with cMyBP-C. This interaction may regulate the binding and unbinding of $\mathrm{CMyBP}-\mathrm{C}$ to myosin proximal subfragment- 2 by initiating rapid phosphorylation of $\mathrm{Ca}^{2+} /$ calmodulin dependent kinase II (CaMKII) targets on MyBP-C or inducing phosphorylation of the RLC by myosin light chain kinase (Lu et al. 2012). The presence of a $\mathrm{Ca}^{2+} /$ calmodulin binding site in the $\mathrm{N}$-terminal region of $\mathrm{cMyBP}-\mathrm{C}$ raises questions about the role of this $\mathrm{Ca}^{2+}$-signalling pathway in each contraction cycle. Does $\mathrm{Ca}^{2+}$ induce a response in cMyBP-C beat to beat, or is there a longer-term integration of the calcium signal by down-stream phosphorylation events?

Another binding partner to MyBP-C is Four and a Half LIM protein 1 (FHL1 or SLIM1), a highly expressed protein in skeletal and cardiac muscle. MyBP-C seems integral for the incorporation of FHL1 into the thick filament (McGrath et al. 2006). While the exact function of FHL1 is unknown, overexpression and knockout results in poor sarcomere assembly. Mutations in muscle LIM protein have been linked to dilated cardiomyopathy (DCM) (Knoll et al. 
2002). Mutations in MyBP-C can therefore have effects downstream through its interactions with these auxiliary proteins.

More recently cardiac formin Fhod3 has been reported to have strong binding interactions $\left(\mathrm{K}_{\mathrm{d}}: 0.8 \mu \mathrm{M}\right)$ with the cMyBP-C (Matsuyama et al. 2018). Like MyBP-C, Fhod3 is localised to the C-zones of the A-band, and MyBP-C is necessary for this localisation. Fhod 3 contributes to actin polymerization, nucleation and recruitment of profilin actin dimers (Blanchoin et al. 2014). It also has an important role in regulating actin assembly in the sarcomere and maintaining cardiac function in perinatal and adult hearts (Ushijima et al. 2018). This further supports the notion that cMyBP-C has multiple roles ranging from structural organisation to the $\mathrm{Ca}^{2+}$ response, and explains why mutations in MyBP-C can have downstream effects in general sarcomere maintenance. For the physiological and structural role of MyBP-C in sarcomere maintenance we direct readers to more specialized reviews of (Flashman et al. 2004; Harris et al. 2011; Moss et al. 2015).

Here we will discuss the current knowledge in the field about MyBP-C and its interactions with other sarcomere proteins. In particular, we will focus on the interplay between
MyBP-C and myosin, MyBP-C and actin and consider the potential complexities that this brings. The inevitable ebb and flow of research activity has led to interest swinging between the MyBP-C interaction with the thick or thin filament. We emphasise here why it is important to bring these together to provide an informed opinion on how this important cardiac regulator functions.

\section{C-terminal interactions of cMyBP-C with myosin}

MyBP-C's location in the sarcomere is limited to the C-zones of the A band (Fig. 2), regularly patterned in 7-9 transverse parallel stripes each containing 3 molecules and approximately $43 \mathrm{~nm}$ apart, which correspond with the axial repeat of myosins along the thick filament (Craig and Offer 1976; Luther et al. 2011). This results in a ratio of MyBP$\mathrm{C}$ : myosin in the C-zone of 1:3. The two C-zones per thick filament cover a third to a half of the thick filament between the ends of the bare zone and the filament tips. This brings up the first unanswered questions about MyBP-C-what

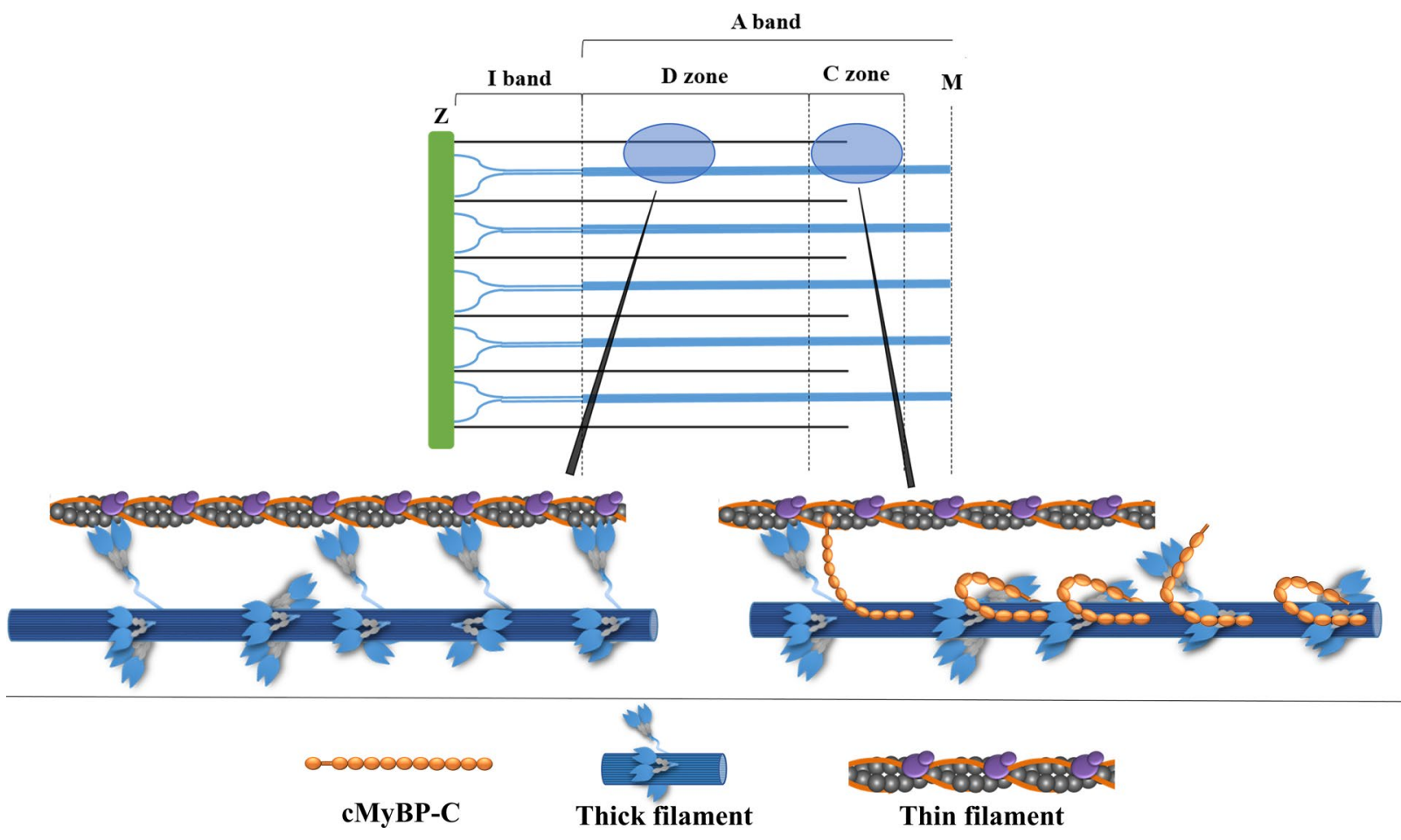

Fig. 2 Schematic diagram of half-sarcomere organisation. The A-band contains thick filaments and overlapping thin filaments, the $\mathrm{C}$-zone is the region of the thick filament with MyBP-C present, and the D-zone has no MyBP-C. The I-band contains thin filaments and also titin, which leads from the Z-line into the thick filaments. These areas are shown with more detail below, highlighting the content of the thick filaments in zone $\mathrm{C}$ versus D. For clarity only every third myosin crown is shown, please refer to Fig. 3 for details on stoichiometry 
structural features confine MyBP-C to this section of the thick filament, and if location is linked to its regulatory role(s), why just this section?

The first identified binding partner of MyBP-C was myo$\sin$, as the name suggests. MyBP-C is anchored to the thick filament through strong binding interactions between the $\mathrm{C} 7$ and C10 domains and the light meromyosin (LMM) and titin backbone (Tonino et al. 2019). This interaction between MyBP-C and titin could explain the restriction of MyBP-C to the C-zone of the thick filament. C10 (of MyBP-C) binds to a short region of LMM (residues 1554-1581 (Human numbering)) through positively charged amino acids (Flashman et al. 2007), but C7-C9 are necessary to maximise binding affinity to between 0.5 and $3.5 \mu \mathrm{M}$ depending on the isoform (Miyamoto et al. 1999; Okagaki et al. 1993) and key for localisation of MyBP-C to the A-band in the sarcomere (Gilbert et al. 1999). Different paralogs of MyBP$\mathrm{C}$ have a higher affinity for the LMM and thick filament in the type of muscle where it is found, i.e. cardiac MyBP-C has higher affinity for cardiac LMM than for skeletal LMM (Alyonycheva et al. 1997).

The focus of the research on the binding properties for the $\mathrm{N}$-terminal domains of MyBP-C have shifted several times over the decades. The $\mathrm{C} 0-\mathrm{C} 7$ domains are thought to extend from the thick filament and able to bind both the heavy meromyosin (HMM) region of the thick filament and the actin filament.

An important aspect of the way in which MyBP-C can regulate the thick filament (and the thin filament) is the 3-D geometrical packing of the proteins into the thick filament. There are 3 MyBP-C for each 9 myosins in the C-zone. Thus, the arrangement is important for how MyBP-C may directly or indirectly interact with each pair of myosin heads (see Fig. 3). As stated above, the repeat pattern of myosins in the thick filament is $43 \mathrm{~nm}$. Within each $43 \mathrm{~nm}$ are 9 myosins and 3 MyBP-C. The myosins are arranged in 3 sets of 3 crowns, $14.3 \mathrm{~nm}$ apart, with each crown rotated $40^{\circ}$ around the thick filament (Zoghbi et al. 2008). The $43 \mathrm{~nm}$ repeat of the MyBP-C stripes therefore corresponds to three MyBP$\mathrm{C}$ molecules at every third crown (Fig. 3a). For MyBP-C to control all myosins in the C-zone it must interact with the myosins in two additional crowns along the filament. MyBP$\mathrm{C}$ affects the packing of myosin heads onto the backbone of the filament and that three crowns cooperate in both packing and activation such that one MyBP-C is sufficient to govern three myosins. EM images of the thick filament suggest possible interactions between the folded myosin heads and the axially adjacent pair of myosin heads away from the bare zone (Woodhead et al. 2005), but higher resolution structures of the thick filament structure are required to establish the detail of such contacts. Given the length of MyBP-C a strong interaction of the $\mathrm{C} 0-\mathrm{C} 2$ domains with a myosin head some distance from the binding site of the $\mathrm{C} 10-\mathrm{C} 7$ domains in the thick filament could sterically restrict additional myosins without the need for a specific binding site.

\section{MyBP-C phosphorylation}

The functional effect of phosphorylation of the cMyBP-C $\mathrm{M}$ domain was first described in intact amphibian muscle (Hartzell 1984). Frog myocardium treated with isoproterenol
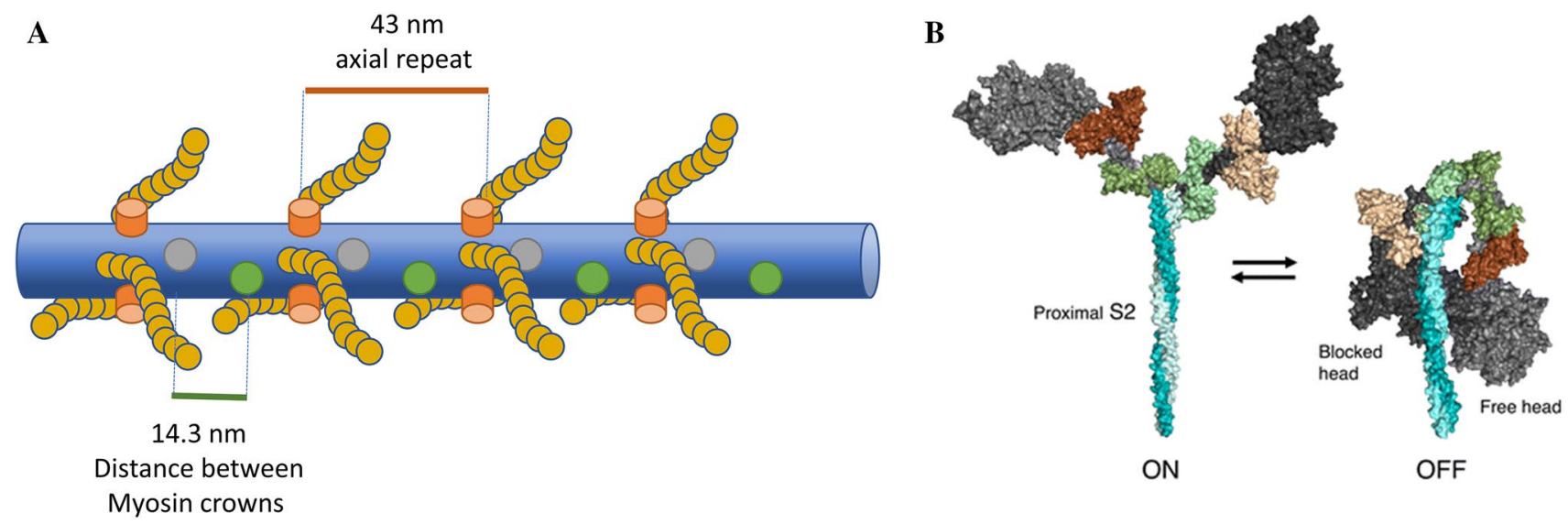

Fig. 3 a The molecular arrangement of the thick filament in the $\mathrm{C}$-zone. Three molecules of MyBP-C associate via their C-terminal regions with myosin $\mathrm{S} 2$ at every third crown (orange cylinders) leading to a $43 \mathrm{~nm}$ spacing between MyBP-C zones which matches the axial repeat. MyBP-C also interacts with myosin heads/neck through the $\mathrm{N}$-terminal regions, although it is clear from the diagram that MyBP-C can possibly extend to other myosins in the $14.3 \mathrm{~nm}$ helical repeat therefore the precise myosin that interacts with MyBP-C is

unknown. Discovering the hinge points of MyBP-C and the geometry of its arrangement on the thick filament will be integral for understanding how MyBP-C works. b Myosin can exist in two clear configurations, left (active. heads-unfolded), right (inactive, heads folded). MyBP-C can modulate these forms of myosin, although the mechanism and location of binding sites are not fully known. Image taken from (Trivedi et al. 2018) 
to up-regulate phosphorylation showed an increase in tension which accelerated contraction and relaxation rates. The serine residues [Ser-273, Ser-282, Ser-302 and Ser307; mouse sequence (Mohamed et al. 1998)] can be phosphorylated by an array of protein kinases, including protein kinase A (PKA) (Gautel et al. 1995; Mohamed et al. 1998), protein kinase C (PKC) (Mohamed et al. 1998), protein kinase D (PKD) (Bardswell et al. 2010), CaMKII (Gautel et al. 1995) and ribosomal S6 kinase (Cuello et al. 2011). cMyBP-C is highly phosphorylated under baseline conditions but phosphorylation is significantly reduced in many cardiac conditions like HCM (Sadayappan et al. 2005). This supports the physiological importance of cMyBP-C phosphorylation. During ischemia, dephosphorylated cMyBP-C was more prone to proteolysis than phosphorylated cMyBPC (Sadayappan et al. 2005) and recently $29 \mathrm{kDa}$ cMyBP-C fragments released during ischemia were shown to be useful early indicators of myocardial infarction (Govindan et al. 2013; Lyngbakken et al. 2019). It is difficult to distinguish the functional effects of phosphorylation of cMyBP-C from other adrenergic targets in the sarcomere, prioritising the use of transgenic models to further enhance our knowledge on these effects. One often used approach is to substitute the serine for aspartate residues to mimic phosphorylation. However it was shown using N-terminal MyBP-C fragments (C1-m-C2) that the functional and structural effects of phosphorylation in interaction with the thin filament are not mimicked by this substitution (Kampourakis et al. 2018). This is a point of consideration when analysing future results and this result will make the experimental study of phosphorylation effects significantly more challenging.

\section{Interactions between the $\mathbf{N}$-terminus of CMyBP-C and myosin}

The sarcomeric arrangement of myosin into thick filaments provides a large interaction area for $\mathrm{cMyBP}-\mathrm{C}$, which is mediated by the $\mathrm{C}$-terminal domains as described above. However, the positioning of the remainder of the molecule is still under debate. Structural data indicates that MyBP-C stretches between the thick and thin filaments (Luther et al. 2011), and measurements of $\mathrm{C} 0$ binding to the regulatory light chain indicate an interaction of the very $\mathrm{N}$ terminus with myosin (Ratti et al. 2011). C1 has also been shown to interact with myosin adjacent to the light chains (Ababou et al. 2008), and C1-C2 binds S2 in a phosphorylation dependent manner (Gruen et al. 1999). These observations clearly suggest that the N-terminus of cMyBP-C modulates myosin, however it is not yet clear how and what is affected. Studies on the mouse myocardium revealed that loss of cMyBP-C resulted in an accelerated stretch activation response consistent with $\mathrm{cMyBP}-\mathrm{C}$ suggesting an acceleration of the cross-bridge kinetics (Stelzer et al. 2006a, c), which was reversed by PKA phosphorylation (Stelzer et al. 2006c). Biochemical studies of isolated proteins suggest an enhancement of the actin activated ATPase at low MyBP-C concentrations that is offset by a greater reduction in ATPase at higher concentrations (Belknap et al. 2014). However, these effects could also be mediated by an interaction with actin, described in more detail below, rather than a direct effect of MyBP-C on myosin. To make the situation more complex, phosphorylation reduces the interaction between cMyBP-C and myosin with inotropic consequences (Gruen et al. 1999; Nag et al. 2017; Toepfer et al. 2013). Such observations would imply that unphosphorylated cMyBP-C inhibits myosin. Yet the basal level of MyBP-C phosphorylation is quite high (Gresham and Stelzer 2016). An interaction between cMyBP-C and a population of the super-relaxed state (SRX) of myosin likely explains this effect. However, the structure of this state still requires a clear definition. The SRX was originally defined as a thick filament state with suppressed ATPase (Hooijman et al. 2011), this does not have a defined structural correlate presently. However, conformations with myosin heads folded back onto one another (Fig. 3b) exist: the J-motif, originally identified in smooth muscle (Wendt et al. 2001) and the Interacting Heads Motif, seen in tarantula (Woodhead et al. 2005), scallop (Stafford et al. 2001) and limulus (Jung et al. 2008) and others (Lee et al. 2018) muscle, and correlate to turned off muscle. Such structures have also been seen in cardiac thick filaments (Zoghbi et al. 2008), suggesting that the SRX may correlate with these such structures. However, the SRX state has been found in HMM and myosin (Nag et al. 2017) suggesting that a state not packed onto the thick filament but still 'off' may exist (Caremani et al. 2019a). Nonetheless these 'off' heads reduce the population of force generating heads, decreasing the maximum tension that can be generated (McNamara et al. 2016; Spudich 2019; Starr and Offer 1978). As long as the number of heads still exceeds the duty cycle requirements then the maximum velocity will be affected to a lesser extent, if at all. Recently the binding site of MyBP-C has been mapped onto myosin in more detail and correlated with HCM mutations in $M Y B P C 3$ to suggest an interaction interface across one face of myosin (the 'mesa') in the ordered relaxed conformation (Nag et al. 2017). Structural studies have shown that myosins leave the 'off' state to form the DRX (disordered relaxed state) state upon phosphorylation of the RLC (Colson et al. 2010; Levine et al. 1996) in striated muscle. These observations echo the effects of RLC phosphorylation on the regulation of tarantula muscle (Brito et al. 2011), and smooth muscle (Lowey and Trybus 2010; Wendt et al. 1999), which are exclusively thick filament regulated muscle types and possess no MyBP-C. However, in striated muscle, formation of the DRX is a compound effect of phosphorylation of the 
RLC (Levine et al. 1996; Stelzer et al. 2006b) and MyBP-C (Colson et al. 2010) as well as temperature and the fraction of the motor in the pre-powerstroke (M.ADP.Pi) conformation. The role and timing of each component is of considerable interest. RLC and MyBP-C phosphorylation appear to result in similar increases in the number of available heads, however lattice spacing appears to be affected more by RLC phosphorylation than effects of MyBP-C (Colson et al. 2007, 2010, 2012; Palmer et al. 2004) (although contested in Sadayappan et al. 2006), suggesting a distinct mechanism of force enhancement.

\section{$\mathrm{N}$-terminal interactions of CMyBP-C with actin}

MyBP-C's interaction with actin was discovered shortly after its ability to bind myosin was determined (Moos et al. 1978). Since then, numerous experimental approaches have collectively shown the N-terminal region of MyBP-C is responsible for these interactions (Belknap et al. 2014; Harris et al. 2016; Inchingolo et al. 2019; Kensler et al. 2011; Lu et al. 2011; Luther et al. 2011; Mun et al. 2011, 2014; Orlova et al. 2011; Risi et al. 2018; Whitten et al. 2008). The N-terminal domains possess some interesting structural attributes; the Ig-like $\mathrm{C} 0$ domain is specific to the cardiac isoform and the proline-alanine rich region between $\mathrm{C} 0$ and $\mathrm{C} 1$ has sequences proposed to bind actin (Squire et al. 2003), that have been linked to modulating contractile velocity across species (Shaffer and Harris 2009). Also at the N-terminus is the M-domain, part of which has been shown to form a tri-helix bundle in isolation or with C2 (Howarth et al. 2012; Michie et al. 2016) and is structurally perturbed by $\mathrm{Ca}^{2+}$-calmodulin binding and phosphorylation (Michie et al. 2016; Previs et al. 2016). The precise regions of binding to actin are likely to vary between isoforms and species (Shaffer et al. 2010; van Dijk et al. 2014), therefore an important current goal is to clarify these observations. Functionally, the role of interaction with actin is becoming clearer from a combination of in vitro and in vivo studies. In vitro motility assays, biochemical ATPase assays and single molecule studies have revealed a cMyBP-C induced sensitization of thin filament activation to calcium consistent with a left shift of the velocity-pCa curve (Belknap et al. 2014; Mun et al. 2014; Previs et al. 2012; Razumova et al. 2006; Saber et al. 2008). However, at high calcium cMyBP-C slowed down actin or thin filament sliding and reduced myosin's ATPase activity. Note, however, that these in vitro assays often use high concentrations of cMyBP-C resulting in high levels of actin saturation. Altogether this suggests a model where cMyBP-C binding at low $\left[\mathrm{Ca}^{2+}\right]$ displaces tropomyosin towards the "closed" position, while at high $\mathrm{Ca}^{2+}$ cMyBP-C blocks or competes with $\mathrm{S} 1$ binding, or creates a viscous load to reduce sliding (Craig et al. 2014; Mun et al. 2014; Walcott et al. 2015). The role of cMyBP-C competing with myosin binding will be different in the sarcomere where the stoichiometry of myosin:cMyBP$\mathrm{C}$ :actin will limit competition between myosin and MyBP-C. Interestingly, in mouse the effects of tropomyosin displacement were highly cMyBP-C domain specific. Only C0C3 caused displacement and increased sensitivity to $\mathrm{Ca}^{2+}$ while shorter N-terminal fragments $(\mathrm{C} 0 \mathrm{C} 1$ and $\mathrm{C} 0 \mathrm{C} 1 \mathrm{f}$ containing the first $17 \mathrm{M}$-domain residues) did show thin filament binding but no effect on the $\mathrm{Ca}^{2+}$ sensitivity and $\mathrm{S} 1$ binding (Belknap et al. 2014; Inchingolo et al. 2019; Mun et al. 2014). This highlights that a clear definition of both long-lived and transient binding sites on actin for cMyBP-C are required.

\section{The governing role of $\mathrm{cMyBP}-\mathrm{C}$}

The role of cMyBP-C is fascinating in its complexity, but its function can potentially be distilled into two actions, governing activation through thin filament interactions, and governing force by controlling myosin head availability through the thick filament. As described above, cMyBP-C is capable of sensitizing the thin filament to myosin binding at low calcium; this has been visualized as a shift in tropomyosin towards the more active 'closed' state (Mun et al. 2011, 2014). However, in these studies, despite visualizing the tropomyosin shift, cMyBP-C was not observed as a clear density instead only the proximal region was visible. Recently, cryo-EM studies were able to visualize the whole of the N-terminal fragment bound to the thin filament (Risi et al. 2018). This difference may be due to the lower levels of decoration used in the negative stain experiments resulting in loss of apparent density due to averaging, or could reflect a dynamic interaction between cMyBP-C and the thin filament; the latter has recently been directly observed (Inchingolo et al. 2019). Imaging fluorescent C0C3 showed a dynamic search along the thin filament, potentially offering a mechanism for sensing its activation state. At high calcium fewer molecules were observed to diffuse, instead binding more strongly, competing with myosin binding and also perhaps providing a viscous load to slow velocity (Inchingolo et al. 2019). These two distinct states, one dynamic and the second static may explain the two binding modes observed using electron microscopy. Indeed, Risi et al. (2018) suggest that $\mathrm{C} 1$ presents a strong interaction with actin further enhanced by $\mathrm{C} 0$, potentially offering a direct structural assignment of these two activities.

Activation of the thin filament provides the trigger to enable contraction, however the force exerted by the myosins is dependent on the number of available heads. This second aspect of cMyBP-C's function is complex and likely involves distinct mechanisms to release heads e.g. through phosphorylation or force-induced. The precise origin of the latter, 
force-induced effects are hotly debated. Driven by x-ray diffraction and fluorescence polarization studies, it remains unclear whether cMyBP-C's function as a force transducer between thick and thin filaments, or simply a modulator of myosin head availability (Caremani et al. 2019b; Fusi et al. 2017; Irving and Craig 2019; Kampourakis et al. 2014; Linari et al. 2015; Reconditi et al. 2017). These findings are further confounded by the recent observation that heads released from the thick filament may not necessarily be in an active state (Caremani et al. 2019a).

Clearly cMyBP-C is an important modulator of cardiac output, at one level working to release heads to increase the force generating capacity of the heart, but then to sequester these heads in a low energy usage state when not required. However, the extent of cooperative force-dependent head release for interaction with the thin filament, and cMyBPC's role in this, is unknown. The differential binding of cMyBP-C N-terminal regions to both myosin and to actin suggests a very important regulatory role for this protein, shuttling between thick and thin filaments, modulated by phosphorylation/load. In a very recent study, this was investigated at multiple levels to demonstrate that hierarchical phosphorylation imbues specific properties on cMyBP-C. At low phosphorylation levels the myosin heads are released and $\mathrm{cMyBP}-\mathrm{C}$ can participate in thin filament activation, whereas at higher phosphorylation levels the activation of actin is blunted, facilitating diastole (Ponnam et al. 2019). This has added a more nuanced appreciation of earlier data showing high levels of phosphorylation decreased the interaction between cMyBP-C and the thin filament (Previs et al. 2016; Shaffer et al. 2009; Weith et al. 2012a, b). However, at high calcium levels the structure of phosphorylated cMyBP-C reverts to one capable of binding the thin filament by direct calcium binding to cMyBP-C (Previs et al. 2016).

These complex interactions make it difficult to tease apart the distinct roles of cMyBP-C, and to understand their importance with the context of the stoichiometric and spatial constraints of the sarcomere. The latter are further exacerbated by the inconsistent alignment between thick and thin filament periodicities (43 and $36 \mathrm{~nm}$, respectively) resulting in clear landing zones for these proteins, as demonstrated for myosin and actin using laser tweezers (Steffen et al. 2001). To more adequately extract an understanding of this complex system will require an appreciation of the activation timescales induced by force, RLC phosphorylation and cMyBP-C phosphorylation. Disentangling this time response may well clarify the roles of thick and thin filament responses to stimulation. In addition, these timescales need to be woven into the thin filament state switching which occurs rapidly, consistent with a similar affinity of TnI for TnC (McKay et al. 1997) in response to $\mathrm{Ca}^{2+}$ and myosin binding (McKillop and Geeves 1993). Phosphorylation of MyBP-C (and other thick and thin filament components) is likely a slower response resulting in a gross change in the contractile capacity of the sarcomere, calcium sensitivity and rates of activation and relaxation. Altogether, these observations highlight the complex role of cMyBP-C in the sarcomere and explain why this protein is such a prolific target of pathogenic mutation.

\section{Does MyBP-C co-orchestrate actomyosin interactions?}

The more we seem to understand about cMyBP-C the more challenging a model is required to describe its function. We have summarised many of the observations in Fig. 4, showing how the relaxed state is a continuum of states capable of modulating the force once the thin filament is activated. The highlighted mechanisms of activation show how the interaction between the thick and thin filaments are inter-dependent and how cMyBP-C lies at the core of this. Since cMyBP$\mathrm{C}$ has the ability to bind both myosin and actin with similar affinities, the mechanisms employed in vivo to regulate its binding partners are crucial to understand (Wang et al. 2016). The stoichiometry and cooperativity of the contacts between cMyBP-C and myosin/actin need to be determined across a range of conditions. This will reveal if release of heads from the thick filament is cooperative and also how titin plays a role in this (presumably as a force conductor). A long-established difference exists between the cooperativity of velocity (e.g. in vitro motility) and tension. The latter has a Hill coefficient typically above 5 and motility is usually below 2. Perhaps the activating effects of cMyBP-C on the thin and thick filaments can reconcile this difference, but how is this regulated? Is there a direct effect of calcium on the thick filament or is this via a different mechanism?

Despite these potential mechanisms of activation, other fundamental questions regarding the localisation of cMyBP$\mathrm{C}$ in the C-zone also need to be addressed. The diversity of cMyBP-C binding partners might suggest a role in sarcomeric organisation and repair, or to offset the calcium gradient in the cardiac sarcomere (Previs et al. 2015). The latter is of interest when considering other muscle types where the T-tubules are located away from the Z-line.

Finally, the molecular picture provided in this review provides a framework that will ultimately require explanation at the physiological contraction level. Such observations show inconsistencies between studies possibly due to the method of study or sample preparation which may affect lattice spacing (Irving and Craig 2019), or inhomogeneities due to phosphorylation between myocyte preparations. To achieve such connectivity on multiple scales will require uniform sample preparation from one source and computational models that scale between such levels (Chase et al. 2004; Mijailovich et al. 2016; Niederer et al. 2019; Tanner et al. 2008; Walcott et al. 2015; Wang et al. 2016). 


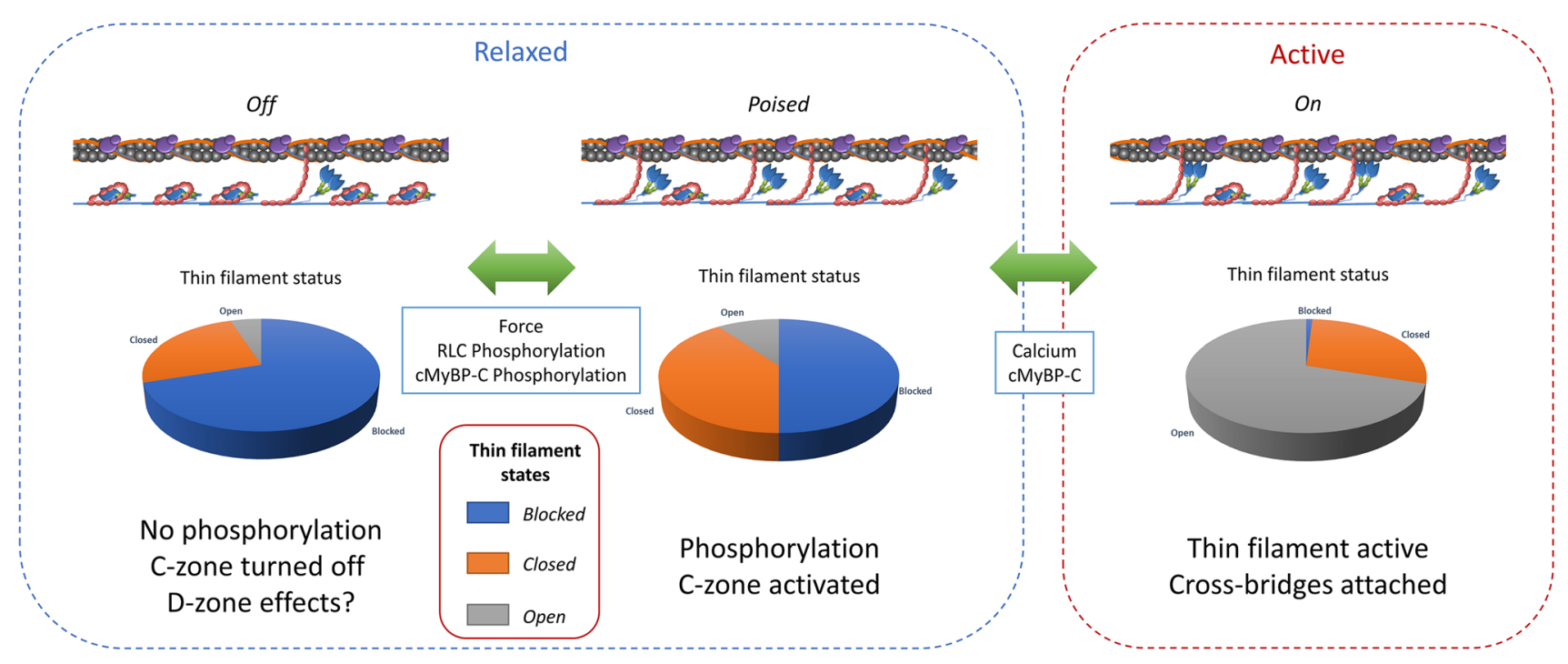

Fig. 4 The interplay between the thick and thin filaments mediated by cMyBP-C. The relaxed state is represented as a continuum of thick filament and thin filament states, which are modulated by phosphorylation and/or force. In a completely relaxed condition very few C-zone heads are likely to be activated, at the other end of the spectrum with RLC, cMyBP-C phosphorylation, and force the number of heads ready for generating tension is maximized. Interestingly, even though phosphorylation of cMyBP-C releases heads from the thick filament, it prevents cMyBP-C interacting with the thin filament. The acute stage of activation, mediated by calcium binding to the thin filament, results in cross-bridge attachment and now cMyBP-C can reveal its second, modulatory role on contraction. The complexity of this process is grossly underestimated in this diagram, however the questions necessary to understand this process are revealed
The continuing story of cMyBP-C is providing a revelation in our understanding of muscle, both in normal and disease conditions. With the advancements in imaging and spectroscopy technologies across a range of resolutions both in vivo and in vitro it is hoped that these can be provide a detailed time-resolved view of cMyBP-C function. Reconciling a stronger molecular understanding with physiological observations using computational methods offers a mechanism to solve the remaining questions of how cMyBP-C modulates contraction and why it is such a prolific site of mutation in disease.

Open Access This article is licensed under a Creative Commons Attribution 4.0 International License, which permits use, sharing, adaptation, distribution and reproduction in any medium or format, as long as you give appropriate credit to the original author(s) and the source, provide a link to the Creative Commons licence, and indicate if changes were made. The images or other third party material in this article are included in the article's Creative Commons licence, unless indicated otherwise in a credit line to the material. If material is not included in the article's Creative Commons licence and your intended use is not permitted by statutory regulation or exceeds the permitted use, you will need to obtain permission directly from the copyright holder. To view a copy of this licence, visit http://creativecommons.org/licenses/by/4.0/.

\section{References}

Ababou A, Rostkova E, Mistry S, Le Masurier C, Gautel M, Pfuhl M (2008) Myosin binding protein C positioned to play a key role in regulation of muscle contraction: structure and interactions of domain C1. J Mol Biol 384:615-630. https://doi.org/10.1016/j. jmb.2008.09.065

Alyonycheva TN, Mikawa T, Reinach FC, Fischman DA (1997) Isoform-specific interaction of the myosin-binding proteins (MyBPs) with skeletal and cardiac myosin is a property of the C-terminal immunoglobulin domain. J Biol Chem 272:2086620872. https://doi.org/10.1074/jbc.272.33.20866

Bardswell SC et al (2010) Distinct sarcomeric substrates are responsible for protein kinase D-mediated regulation of cardiac myofilament $\mathrm{Ca}^{2+}$ sensitivity and cross-bridge cycling. J Biol Chem 285:5674-5682. https://doi.org/10.1074/jbc.M109.066456

Belknap B, Harris SP, White HD (2014) Modulation of thin filament activation of myosin ATP hydrolysis by N-terminal domains of cardiac myosin binding protein-C. Biochemistry 53:6717-6724. https://doi.org/10.1021/bi500787f

Blanchoin L, Boujemaa-Paterski R, Sykes C, Plastino J (2014) Actin dynamics, architecture, and mechanics in cell motility. Physiol Rev 94:235-263. https://doi.org/10.1152/physrev.00018.2013

Bonne $\mathrm{G}$ et al (1995) Cardiac myosin binding protein-C gene splice acceptor site mutation is associated with familial hypertrophic cardiomyopathy. Nat Genet 11:438-440. https://doi.org/10.1038/ng1295-438

Brito R et al (2011) A molecular model of phosphorylation-based activation and potentiation of tarantula muscle thick filaments. J Mol Biol 414:44-61. https://doi.org/10.1016/j.jmb.2011.09.017

Caremani M et al (2019a) Low temperature traps myosin motors of mammalian muscle in a refractory state that prevents activation. J Gen Physiol. https://doi.org/10.1085/jgp.201912424

Caremani $\mathrm{M}$ et al (2019b) Inotropic interventions do not change the resting state of myosin motors during cardiac diastole. J Gen Physiol 151:53-65. https://doi.org/10.1085/jgp.201812196

Carrier L, Mearini G, Stathopoulou K, Cuello F (2015) Cardiac myosin-binding protein C (MYBPC3) in cardiac pathophysiology. Gene 573:188-197. https://doi.org/10.1016/j.gene.2015.09.008 
Chase PB, Macpherson JM, Daniel TL (2004) A spatially explicit nanomechanical model of the half-sarcomere: myofilament compliance affects $\mathrm{Ca}(2+)$-activation. Ann Biomed Eng 32:1559-1568. https://doi.org/10.1114/b:abme.0000049039.89173.08

Colson BA, Bekyarova T, Fitzsimons DP, Irving TC, Moss RL (2007) Radial displacement of myosin cross-bridges in mouse myocardium due to ablation of myosin binding protein-C. J Mol Biol 367:36-41. https://doi.org/10.1016/j.jmb.2006.12.063

Colson BA, Locher MR, Bekyarova T, Patel JR, Fitzsimons DP, Irving TC, Moss RL (2010) Differential roles of regulatory light chain and myosin binding protein-C phosphorylations in the modulation of cardiac force development. J Physiol 588:981-993. https ://doi.org/10.1113/jphysiol.2009.183897

Colson BA et al (2012) Myosin binding protein-C phosphorylation is the principal mediator of protein kinase A effects on thick filament structure in myocardium. J Mol Cell Cardiol 53:609-616. https://doi.org/10.1016/j.yjmcc.2012.07.012

Craig R, Offer G (1976) The location of C-protein in rabbit skeletal muscle. Proc R Soc Lond B 192:451-461

Craig R, Lee KH, Mun JY, Torre I, Luther PK (2014) Structure, sarcomeric organization, and thin filament binding of cardiac myosin-binding protein-C. Pflugers Arch 466:425-431. https ://doi.org/10.1007/s00424-013-1426-6

Cuello F, Bardswell SC, Haworth RS, Ehler E, Sadayappan S, Kentish JC, Avkiran M (2011) Novel role for p90 ribosomal S6 kinase in the regulation of cardiac myofilament phosphorylation. J Biol Chem 286:5300-5310. https://doi.org/10.1074/jbc. M110.202713

Flashman E, Redwood C, Moolman-Smook J, Watkins H (2004) Cardiac myosin binding protein $\mathrm{C}$ : its role in physiology and disease. Circ Res 94:1279-1289. https://doi.org/10.1161/01. RES.0000127175.21818.C2

Flashman E, Watkins H, Redwood C (2007) Localization of the binding site of the C-terminal domain of cardiac myosin-binding protein-C on the myosin rod. Biochem J 401:97-102. https:// doi.org/10.1042/BJ20060500

Fusi L et al (2017) Minimum number of myosin motors accounting for shortening velocity under zero load in skeletal muscle. J Physiol 595:1127-1142. https://doi.org/10.1113/JP273299

Gautel M, Zuffardi O, Freiburg A, Labeit S (1995) Phosphorylation switches specific for the cardiac isoform of myosin binding protein-C: a modulator of cardiac contraction? EMBO J 14:1952-1960

Geeves MA, Holmes KC (1999) Structural mechanism of muscle contraction AnnuRevBiochem 68:687-728

Gilbert R, Cohen JA, Pardo S, Basu A, Fischman DA (1999) Identification of the A-band localization domain of myosin binding proteins $\mathrm{C}$ and $\mathrm{H}$ (MyBP-C, MyBP-H) in skeletal muscle. $\mathrm{J}$ Cell Sci 112(Pt 1):69-79

Govindan $\mathrm{S}$ et al (2013) Increase in cardiac myosin binding protein-C plasma levels is a sensitive and cardiac-specific biomarker of myocardial infarction Am J. Cardiovasc Dis 3:60-70

Gresham KS, Stelzer JE (2016) The contributions of cardiac myosin binding protein $\mathrm{C}$ and troponin I phosphorylation to betaadrenergic enhancement of in vivo cardiac function. J Physiol 594:669-686. https://doi.org/10.1113/JP270959

Gruen M, Prinz H, Gautel M (1999) cAPK-phosphorylation controls the interaction of the regulatory domain of cardiac myosin binding protein $\mathrm{C}$ with myosin-S2 in an on-off fashion. FEBS Lett 453:254-259

Harris SP et al (2002) Hypertrophic cardiomyopathy in cardiac myosin binding protein-C knockout mice. Circ Res 90:594-601

Harris SP, Lyons RG, Bezold KL (2011) the thick of it: HCM-causing mutations in myosin binding proteins of the thick filament. Circ Res 108:751-764. https://doi.org/10.1161/CIRCRESAHA .110 .231670
Harris SP, Belknap B, Van Sciver RE, White HD, Galkin VE (2016) $\mathrm{C} 0$ and $\mathrm{C} 1 \mathrm{~N}$-terminal Ig domains of myosin binding protein $\mathrm{C}$ exert different effects on thin filament activation. Proc Natl Acad Sci USA 113:1558-1563. https://doi.org/10.1073/ pnas. 1518891113

Hartzell HC (1984) Phosphorylation of C-protein in intact amphibian cardiac muscle. Correlation between 32P incorporation and twitch relaxation. J Gen Physiol 83:563-588. https://doi. org/10.1085/jgp.83.4.563

Hartzell HC, Sale WS (1985) Structure of C protein purified from cardiac muscle. J Cell Biol 100:208-215. https://doi.org/10.1083/ jcb.100.1.208

Hartzell HC, Titus L (1982) Effects of cholinergic and adrenergic agonists on phosphorylation of a 165,000-dalton myofibrillar protein in intact cardiac muscle. J Biol Chem 257:2111-2120

Harvey PA, Leinwand LA (2011) The cell biology of disease: cellular mechanisms of cardiomyopathy. J Cell Biol 194:355-365. https://doi.org/10.1083/jcb.201101100

Hooijman P, Stewart MA, Cooke R (2011) A new state of cardiac myosin with very slow ATP turnover: a potential cardioprotective mechanism in the heart. Biophys J 100:1969-1976. https ://doi.org/10.1016/j.bpj.2011.02.061

Howarth JW, Ramisetti S, Nolan K, Sadayappan S, Rosevear PR (2012) Structural insight into unique cardiac myosin-binding protein-C motif: a partially folded domain. J Biol Chem 287:8254-8262. https://doi.org/10.1074/jbc.M111.309591

Inchingolo AV, Previs SB, Previs MJ, Warshaw DM, Kad NM (2019) Revealing the mechanism of how cardiac myosin-binding protein $\mathrm{C} \mathrm{N}$-terminal fragments sensitize thin filaments for myosin binding. Proc Natl Acad Sci USA 116:6828-6835. https://doi. org/10.1073/pnas.1816480116

Irving TC, Craig R (2019) Getting into the thick (and thin) of it. J Gen Physiol 151:610-613. https://doi.org/10.1085/jgp.20181 2307

Jung HS, Komatsu S, Ikebe M, Craig R (2008) Head-head and headtail interaction: a general mechanism for switching off myosin II activity in cells. Mol Biol Cell 19:3234-3242. https://doi. org/10.1091/mbc.E08-02-0206

Kampourakis T, Yan Z, Gautel M, Sun YB, Irving M (2014) Myosin binding protein-C activates thin filaments and inhibits thick filaments in heart muscle cells. Proc Natl Acad Sci USA 111:1876318768. https://doi.org/10.1073/pnas. 1413922112

Kampourakis T, Ponnam S, Sun YB, Sevrieva I, Irving M (2018) Structural and functional effects of myosin-binding protein-C phosphorylation in heart muscle are not mimicked by serine-toaspartate substitutions. J Biol Chem 293:14270-14275. https:// doi.org/10.1074/jbc.AC118.004816

Kensler RW, Shaffer JF, Harris SP (2011) Binding of the N-terminal fragment C0-C2 of cardiac MyBP-C to cardiac F-actin. J Struct Biol 174:44-51. https://doi.org/10.1016/j.jsb.2010.12.003

Knoll R, Hoshijima M, Chien KR (2002) Z-line proteins: implications for additional functions. Eur Heart J Suppl 4:I13-I17. https://doi. org/10.1016/S1520-765x(02)90105-7

Lee $\mathrm{KH}$ et al (2018) Interacting-heads motif has been conserved as a mechanism of myosin II inhibition since before the origin of animals. Proc Natl Acad Sci USA 115:E1991-E2000. https://doi. org/10.1073/pnas.1715247115

Levine RJ, Kensler RW, Yang Z, Stull JT, Sweeney HL (1996) Myosin light chain phosphorylation affects the structure of rabbit skeletal muscle thick filaments. Biophys J 71:898-907. https://doi. org/10.1016/S0006-3495(96)79293-7

Linari M et al (2015) Force generation by skeletal muscle is controlled by mechanosensing in myosin filaments. Nature 528:276-279. https://doi.org/10.1038/nature15727 
Lowey S, Trybus KM (2010) Common structural motifs for the regulation of divergent class II myosins. J Biol Chem 285:1640316407. https://doi.org/10.1074/jbc.R109.025551

Lu YL, Kwan AH, Trewhella J, Jeffries CM (2011) The C0C1 fragment of human cardiac myosin binding protein $\mathrm{C}$ has common binding determinants for both actin and myosin. J Mol Biol 413:908-913. https://doi.org/10.1016/j.jmb.2011.09.026

Lu Y, Kwan AH, Jeffries CM, Guss JM, Trewhella J (2012) The motif of human cardiac myosin-binding protein $\mathrm{C}$ is required for its $\mathrm{Ca}^{2+}$-dependent interaction with calmodulin. J Biol Chem 287:31596-31607. https://doi.org/10.1074/jbc.M112.383299

Luther PK et al (2011) Direct visualization of myosin-binding protein $\mathrm{C}$ bridging myosin and actin filaments in intact muscle. Proc Natl Acad Sci USA 108:11423-11428. https://doi.org/10.1073/ pnas. 1103216108

Lyngbakken MN, Myhre PL, Rosjo H, Omland T (2019) Novel biomarkers of cardiovascular disease: applications in clinical practice. Crit Rev Clin Lab Sci 56:33-60. https://doi. org/10.1080/10408363.2018.1525335

Maron BJ, Maron MS (2013) Hypertrophic cardiomyopathy. Lancet 381:242-255. https://doi.org/10.1016/S0140-6736(12)60397-3

Matsuyama $S$ et al (2018) Interaction between cardiac myosinbinding protein $\mathrm{C}$ and formin Fhod3. Proc Natl Acad Sci USA 115:E4386-E4395. https://doi.org/10.1073/pnas.1716498115

McGrath MJ et al (2006) Four and a half LIM protein 1 binds myosinbinding protein $\mathrm{C}$ and regulates myosin filament formation and sarcomere assembly. J Biol Chem 281:7666-7683. https://doi. org/10.1074/jbc.M512552200

McKay RT, Tripet BP, Hodges RS, Sykes BD (1997) Interaction of the second binding region of troponin I with the regulatory domain of skeletal muscle troponin $\mathrm{C}$ as determined by NMR spectroscopy. J Biol Chem 272:28494-28500. https://doi.org/10.1074/ jbc.272.45.28494

McKillop DF, Geeves MA (1993) Regulation of the interaction between actin and myosin subfragment 1: evidence for three states of the thin filament. Biophys J 65:693-701. https://doi. org/10.1016/S0006-3495(93)81110-X

McNamara JW et al (2016) Ablation of cardiac myosin binding protein$\mathrm{C}$ disrupts the super-relaxed state of myosin in murine cardiomyocytes. J Mol Cell Cardiol 94:65-71. https://doi.org/10.1016/j. yjmcc.2016.03.009

Michie KA, Kwan AH, Tung CS, Guss JM, Trewhella J (2016) A highly conserved yet flexible linker is part of a polymorphic protein-binding domain in myosin-binding protein $\mathrm{C}$ structure 24:2000-2007. https://doi.org/10.1016/j.str.2016.08.018

Mijailovich SM, Kayser-Herold O, Stojanovic B, Nedic D, Irving TC, Geeves MA (2016) Three-dimensional stochastic model of actinmyosin binding in the sarcomere lattice. J Gen Physiol 148:459488. https://doi.org/10.1085/jgp.201611608

Miyamoto CA, Fischman DA, Reinach FC (1999) The interface between MyBP-C and myosin: site-directed mutagenesis of the CX myosin-binding domain of MyBP-C. J Muscle Res Cell Motil 20:703-715. https://doi.org/10.1023/a:1005513312939

Mohamed AS, Dignam JD, Schlender KK (1998) Cardiac myosin-binding protein $\mathrm{C}$ (MyBP-C): identification of protein kinase $\mathrm{A}$ and protein kinase $\mathrm{C}$ phosphorylation sites. Arch Biochem Biophys 358:313-319. https://doi.org/10.1006/abbi.1998.0857

Moos C, Mason CM, Besterman JM, Feng IN, Dubin JH (1978) The binding of skeletal muscle C-protein to F-actin, and its relation to the interaction of actin with myosin subfragment-1. J Mol Biol 124:571-586. https://doi.org/10.1016/0022-2836(78)90172-9

Moss RL, Fitzsimons DP, Ralphe JC (2015) Cardiac MyBP-C regulates the rate and force of contraction in mammalian myocardium. Circ Res 116:183-192. https://doi.org/10.1161/CIRCRESAHA.116.300561

Mun JY, Gulick J, Robbins J, Woodhead J, Lehman W, Craig R (2011) Electron microscopy and $3 \mathrm{D}$ reconstruction of F-actin decorated with cardiac myosin-binding protein C (cMyBP-C). J Mol Biol 410:214-225. https://doi.org/10.1016/j.jmb.2011.05.010

Mun JY et al (2014) Myosin-binding protein C displaces tropomyosin to activate cardiac thin filaments and governs their speed by an independent mechanism. Proc Natl Acad Sci USA 111:21702175. https://doi.org/10.1073/pnas.1316001111

Nag S et al (2017) The myosin mesa and the basis of hypercontractility caused by hypertrophic cardiomyopathy mutations. Nat Struct Mol Biol 24:525-533. https://doi.org/10.1038/nsmb.3408

Niederer SA, Campbell KS, Campbell SG (2019) A short history of the development of mathematical models of cardiac mechanics. J Mol Cell Cardiol 127:11-19. https://doi.org/10.1016/j.yjmcc 2018.11.015

Okagaki T, Weber FE, Fischman DA, Vaughan KT, Mikawa T, Reinach FC (1993) The major myosin-binding domain of skeletal muscle MyBP-C (C protein) resides in the $\mathrm{COOH}$-terminal, immunoglobulin C2 motif. J Cell Biol 123:619-626. https:// doi.org/10.1083/jcb.123.3.619

Orlova A, Galkin VE, Jeffries CMJ, Egelman EH, Trewhella J (2011) The $\mathrm{N}$-terminal domains of myosin binding protein $\mathrm{C}$ can bind polymorphically to F-actin. J Mol Biol 412:379-386. https:// doi.org/10.1016/j.jmb.2011.07.056

Palmer BM et al (2004) Reduced cross-bridge dependent stiffness of skinned myocardium from mice lacking cardiac myosin binding protein-C. Mol Cell Biochem 263:73-80. https://doi. org/10.1023/B:Mcbi.0000041849.60591.45

Ponnam S, Sevrieva I, Sun YB, Irving M, Kampourakis T (2019) Site-specific phosphorylation of myosin binding protein-C coordinates thin and thick filament activation in cardiac muscle. Proc Natl Acad Sci USA 116:15485-15494. https://doi. org/10.1073/pnas.1903033116

Previs MJ, Beck Previs S, Gulick J, Robbins J, Warshaw DM (2012) Molecular mechanics of cardiac myosin-binding protein $\mathrm{C}$ in native thick filaments. Science 337:1215-1218. https://doi. org/10.1126/science. 1223602

Previs MJ et al (2015) Myosin-binding protein C corrects an intrinsic inhomogeneity in cardiac excitation-contraction coupling. Sci Adv. https://doi.org/10.1126/sciadv.1400205

Previs MJ et al (2016) Phosphorylation and calcium antagonistically tune myosin-binding protein C's structure and function. Proc Natl Acad Sci USA 113:3239-3244. https://doi.org/10.1073/ pnas. 1522236113

Ratti J, Rostkova E, Gautel M, Pfuhl M (2011) Structure and interactions of myosin-binding protein $\mathrm{C}$ domain $\mathrm{C} 0$ : cardiac-specific regulation of myosin at its neck? J Biol Chem 286:12650 12658. https://doi.org/10.1074/jbc.M110.156646

Razumova MV, Shaffer JF, Tu AY, Flint GV, Regnier M, Harris SP (2006) Effects of the N-terminal domains of myosin binding protein-C in an in vitro motility assay: evidence for long-lived cross-bridges. J Biol Chem 281:35846-35854. https://doi. org/10.1074/jbc.M606949200

Reconditi M et al (2017) Myosin filament activation in the heart is tuned to the mechanical task. Proc Natl Acad Sci USA 114:3240-3245. https://doi.org/10.1073/pnas.1619484114

Risi C, Belknap B, Forgacs-Lonart E, Harris SP, Schroder GF, White HD, Galkin VE (2018) N-terminal domains of cardiac myosin binding protein $\mathrm{C}$ cooperatively activate the thin filament. Structure 26(1604-1611):e1604. https://doi.org/10.1016/j. str.2018.08.007

Saber W, Begin KJ, Warshaw DM, VanBuren P (2008) Cardiac myosin binding protein- $\mathrm{C}$ modulates actomyosin binding and kinetics in the in vitro motility assay. $\mathrm{J}$ Mol Cell Cardiol 44:1053-1061. https://doi.org/10.1016/j.yjmcc.2008.03.012

Sadayappan S et al (2005) Cardiac myosin-binding protein-C phosphorylation and cardiac function. Circ Res 97:1156-1163. https://doi.org/10.1161/01.RES.0000190605.79013.4d 
Sadayappan S et al (2006) Cardiac myosin binding protein C phosphorylation is cardioprotective. Proc Natl Acad Sci USA 103:16918-16923. https://doi.org/10.1073/pnas.0607069103

Semsarian C, Ingles J, Maron MS, Maron BJ (2015) New perspectives on the prevalence of hypertrophic cardiomyopathy. $\mathrm{J}$ Am Coll Cardiol 65:1249-1254. https://doi.org/10.1016/j. jacc.2015.01.019

Shaffer JF, Harris SP (2009) Species-specific differences in the ProAla rich region of cardiac myosin binding protein-C. J Muscle Res Cell Motil 30:303-306. https://doi.org/10.1007/s1097 4-010-9207-8

Shaffer JF, Kensler RW, Harris SP (2009) The myosin-binding protein $\mathrm{C}$ motif binds to $\mathrm{F}$-actin in a phosphorylation-sensitive manner. J Biol Chem 284:12318-12327. https://doi. org/10.1074/jbc.M808850200

Shaffer JF, Wong P, Bezold KL, Harris SP (2010) Functional differences between the $\mathrm{N}$-terminal domains of mouse and human myosin binding protein-C. J Biomed Biotechnol 2010:789798. https://doi.org/10.1155/2010/789798

Spudich JA (2019) Three perspectives on the molecular basis of hypercontractility caused by hypertrophic cardiomyopathy mutations. Pflugers Arch 471:701-717. https://doi.org/10.1007/s00424-01902259-2

Squire JM, Luther PK, Knupp C (2003) Structural evidence for the interaction of C-protein (MyBP-C) with actin and sequence identification of a possible actin-binding domain. J Mol Biol 331:713-724. https://doi.org/10.1016/s0022-2836(03)00781-2

Stafford WF, Jacobsen MP, Woodhead J, Craig R, O’Neall-Hennessey E, Szent-Gyorgyi AG (2001) Calcium-dependent structural changes in scallop heavy meromyosin. J Mol Biol 307:137-147. https://doi.org/10.1006/jmbi.2000.4490

Starr R, Offer G (1971) Polypeptide chains of intermediate molecular weight in myosin preparations. FEBS Lett 15:40-44

Starr R, Offer G (1978) The interaction of C-protein with heavy meromyosin and subfragment-2. Biochem J 171:813-816

Steffen W, Smith D, Simmons R, Sleep J (2001) Mapping the actin filament with myosin. Proc Natl Acad Sci USA 98:14949-14954

Stelzer JE, Dunning SB, Moss RL (2006a) Ablation of cardiac myosin-binding protein- $\mathrm{C}$ accelerates stretch activation in murine skinned myocardium. Circ Res 98:1212-1218. https://doi. org/10.1161/01.RES.0000219863.94390.ce

Stelzer JE, Patel JR, Moss RL (2006b) Acceleration of stretch activation in murine myocardium due to phosphorylation of myosin regulatory light chain. J Gen Physiol 128:261-272. https://doi. org/10.1085/jgp.200609547

Stelzer JE, Patel JR, Moss RL (2006c) Protein kinase A-mediated acceleration of the stretch activation response in murine skinned myocardium is eliminated by ablation of cMyBP-C. Circul Res 99:884-890. https://doi.org/10.1161/01.Res.0000245191.34690 .66

Tanner BC, Regnier M, Daniel TL (2008) A spatially explicit model of muscle contraction explains a relationship between activation phase, power and ATP utilization in insect flight. J Exp Biol 211:180-186. https://doi.org/10.1242/jeb.013466

Toepfer C et al (2013) Myosin regulatory light chain (RLC) phosphorylation change as a modulator of cardiac muscle contraction in disease. J Biol Chem 288:13446-13454. https://doi.org/10.1074/ jbc.M113.455444

Tonino P, Kiss B, Gohlke J, Smith JE 3rd, Granzier H (2019) Fine mapping titin's C-zone: matching cardiac myosin-binding protein $\mathrm{C}$ stripes with titin's super-repeats. J Mol Cell Cardiol 133:47-56. https://doi.org/10.1016/j.yjmcc.2019.05.026

Trivedi DV, Adhikari AS, Sarkar SS et al (2018) Hypertrophic cardiomyopathy and the myosin mesa: viewing an old disease in a new light. Biophys Rev. https://doi.org/10.1007/s12551-017-0274-6

Ushijima T, Fujimoto N, Matsuyama S et al (2018) The actin-organizing formin protein Fhod3 is required for postnatal development and functional maintenance of the adult heart in mice. J Biol Chem. https://doi.org/10.1074/jbc.M117.813931

van Dijk SJ, Bezold KL, Harris SP (2014) Earning stripes: myosin binding protein-C interactions with actin. Pflugers Arch 466:445-450. https://doi.org/10.1007/s00424-013-1432-8

Walcott S, Docken S, Harris SP (2015) Effects of cardiac Myosin binding protein-C on actin motility are explained with a dragactivation-competition model. Biophys J 108:10-13. https://doi. org/10.1016/j.bpj.2014.11.1852

Wang C, Schwan J, Campbell SG (2016) Slowing of contractile kinetics by myosin-binding protein $\mathrm{C}$ can be explained by its cooperative binding to the thin filament. J Mol Cell Cardiol 96:2-10. https://doi.org/10.1016/j.yjmcc.2015.10.007

Watkins $\mathrm{H}$ et al (1995) Mutations in the cardiac myosin binding protein-C gene on chromosome 11 cause familial hypertrophic cardiomyopathy. Nat Genet 11:434-437. https://doi.org/10.1038/ ng 1295-434

Weber FE, Vaughan KT, Reinach FC, Fischman DA (1993) Complete sequence of human fast-type and slow-type muscle myosinbinding-protein $\mathrm{C}$ (MyBP-C). Differential expression, conserved domain structure and chromosome assignment. Eur J Biochem 216:661-669. https://doi.org/10.1111/j.1432-1033.1993.tb181 $86 . \mathrm{x}$

Weith A, Sadayappan S, Gulick J, Previs MJ, Vanburen P, Robbins J, Warshaw DM (2012a) Unique single molecule binding of cardiac myosin binding protein- $\mathrm{C}$ to actin and phosphorylation-dependent inhibition of actomyosin motility requires 17 amino acids of the motif domain. J Mol Cell Cardiol 52:219-227. https://doi. org/10.1016/j.yjmcc.2011.09.019

Weith AE, Previs MJ, Hoeprich GJ, Previs SB, Gulick J, Robbins J, Warshaw DM (2012b) The extent of cardiac myosin binding protein-C phosphorylation modulates actomyosin function in a graded manner. J Muscle Res Cell Motil 33:449-459. https://doi. org/10.1007/s10974-012-9312-y

Wendt T, Taylor D, Messier T, Trybus KM, Taylor KA (1999) Visualization of head-head interactions in the inhibited state of smooth muscle myosin. J Cell Biol 147:1385-1390

Wendt T, Taylor D, Trybus KM, Taylor K (2001) Three-dimensional image reconstruction of dephosphorylated smooth muscle heavy meromyosin reveals asymmetry in the interaction between myosin heads and placement of subfragment 2. Proc Natl Acad Sci USA 98:4361-4366. https://doi.org/10.1073/pnas.071051098

Whitten AE, Jeffries CM, Harris SP, Trewhella J (2008) Cardiac myosin-binding protein $\mathrm{C}$ decorates F-actin: implications for cardiac function. Proc Natl Acad Sci USA 105:18360-18365. https://doi. org/10.1073/pnas.0808903105

Woodhead JL, Zhao FQ, Craig R, Egelman EH, Alamo L, Padron R (2005) Atomic model of a myosin filament in the relaxed state. Nature 436:1195-1199. https://doi.org/10.1038/nature03920

Yamamoto K, Moos C (1983) The C-proteins of rabbit red, white, and cardiac muscles. J Biol Chem 258:8395-8401

Yasuda M, Koshida S, Sato N, Obinata T (1995) Complete primary structure of chicken cardiac C-protein (MyBP-C) and its expression in developing striated muscles. J Mol Cell Cardiol 27:2275-2286

Zoghbi ME, Woodhead JL, Moss RL, Craig R (2008) Three-dimensional structure of vertebrate cardiac muscle myosin filaments. Proc Natl Acad Sci USA 105:2386-2390. https://doi. org/10.1073/pnas.0708912105

Publisher's Note Springer Nature remains neutral with regard to jurisdictional claims in published maps and institutional affiliations. 